Carnets de géographes

GÉOGRAPHES

$3 \mid 2011$

Les géographies des enfants et des jeunes

\title{
Les Mapuches du Chili : des représentations aux pratiques de l'espace. Géographie(s) d'un territoire autochtone
}

Bastien Sepúlveda

\section{OpenEdition}

Journals

Édition électronique

URL : http://journals.openedition.org/cdg/2561

DOI : $10.4000 / \mathrm{cdg} .2561$

ISSN : 2107-7266

Éditeur

UMR 245 - CESSMA

Référence électronique

Bastien Sepúlveda, «Les Mapuches du Chili : des représentations aux pratiques de l'espace.

Géographie(s) d'un territoire autochtone », Carnets de géographes [En ligne], 3 | 2011, mis en ligne le 01 décembre 2011, consulté le 23 septembre 2020. URL : http://journals.openedition.org/cdg/2561 ; DOI : https://doi.org/10.4000/cdg.2561

Ce document a été généré automatiquement le 23 septembre 2020.

\section{(†) $\Theta$}

La revue Carnets de géographes est mise à disposition selon les termes de la Licence Creative Commons Attribution - Pas d'Utilisation Commerciale - Pas de Modification 4.0 International. 


\title{
Les Mapuches du Chili : des représentations aux pratiques de l'espace. Géographie(s) d'un territoire autochtone
}

\author{
Bastien Sepúlveda
}

1 Menée à partir d'enquêtes réalisées chez les Mapuches du Chili, cette thèse propose une approche géographique de la question autochtone et sa résurgence dans le débat public en Amérique Latine. Elle s'intéresse aux différentes formes de contestation de l'Etat auquel les dirigeants des mouvements indianistes s'adressent en revendiquant un territoire dont ils estiment avoir été dépossédés. Les discours sur le territoire et ses représentations sont examinés dans un exercice de déconstruction d'un imaginaire géographique ancré dans des communautés rurales dites «traditionnelles» et idéalisées comme lieu de reproduction d'une culture sur laquelle l'histoire n'aurait pas prise. S'appuyant sur un travail de terrain mené entre ces mêmes communautés et les grands centres urbains du pays vers lesquels convergent aujourd'hui d'importants flux migratoires, la thèse montre l'écart considérable séparant les territorialités professées des territorialités vécues. Une explication à cet écart se trouve dans l'influence que l'Etat exerce sur les manières de concevoir et de se représenter le territoire par le maintien d'une certaine forme de domination coloniale à l'égard des peuples autochtones. Ce faisant, la thèse tente de démontrer qu'il n'existe donc pas une mais plusieurs lectures d'un même espace qui, combinées, permettent de poser les bases d'une géographie du territoire mapuche.

2 Partant d'une mise en perspective des luttes autochtones dans le contexte latinoaméricain, le mémoire explore de manière détaillée la nature des liens tissés entre territoire et autochtonie chez les Mapuches du Chili. Un premier axe de lecture est ainsi tracé dans le sillage du traitement donné par l'Etat chilien à la question territoriale mapuche au cours de l'histoire. Un intérêt particulier est porté notamment aux fondements de l'ordre géographique imposé dans le centre-sud du pays au 
lendemain de la campagne militaire de Pacification de l'Araucanie (1861-1881). En permettant au Chili de ceindre ce que ses classes dirigeantes considéraient depuis toujours comme son étendue naturelle, l'annexion de l'actuelle région d'Araucanie ouvrit une manne foncière essentiellement destinée à la production de blé pour l'exportation. Cette configuration particulière explique sans aucun doute pourquoi la question territoriale mapuche a longtemps été traitée comme un simple problème de terres. Si, récemment, les politiques indigénistes se sont davantage centrées sur des questions de développement, il faut comprendre une telle inflexion comme une réponse ou une réaction à la résurgence des mobilisations mapuches dans le contexte post-dictatorial ${ }^{1}$.

3 Considéré aujourd'hui comme l'un des mouvements autochtones les plus actifs à l'échelle continentale, le mouvement mapuche est aussi et surtout le premier à avoir vu le jour depuis la fondation de l'Etat moderne en Amérique Latine. C'est effectivement en Araucanie, dans les années ayant suivi la défaite politico-militaire mapuche, que sont apparues les premières organisations autochtones du continent. Tout en contextualisant l'émergence de ces organisations et en identifiant, à ce titre, différents cycles de revendications, la thèse porte une attention particulière aux contenus des discours formulés par les leaders indianistes, notamment en ce qui réfère au territoire. C'est là un second axe de lecture qui, articulé sur les territorialités professées par ces leaders, propose une géographie du territoire mapuche alternative à celle de l'ordre colonial. Propulsé au cœur du processus de construction nationale mapuche, le territoire revendiqué dessine ainsi les contours d'une géographie subversive, née de ce qu'il convient d'appeler le tournant postcolonial mapuche.

Mais en puisant dans l'histoire les éléments permettant de légitimer leurs demandes contemporaines, les leaders autochtones produisent ce que Denis Retaillé nomme un " anachronisme géographique », dans le sens où l'Araucanie d'aujourd'hui et celle de la période antérieure à la "pacification " ne sont pas des espaces situés dans le même temps. La société mapuche contemporaine ne tient effectivement pas ou plus dans le cadre territorial revendiqué par les dirigeants autochtones qui, dans leurs discours, exaltent les traditions et la vie en communauté dans les terroirs d'origine. Soumises à un violent processus de mise en réduction, lesdites communautés sont devenues le théâtre d'importantes restructurations territoriales, en même temps que le point d'ancrage de vastes réseaux migratoires faisant voler en éclat toute tentative de définition de l'identité mapuche par un lien essentiel à la terre et au terroir. Dans un contexte de crise des agricultures de subsistance, nombreux sont les Mapuches ayant dû migrer vers les grands centres urbains du pays, tout au long du XXe siècle. Aussi, d'après le dernier recensement réalisé en 2002, près de $65 \%$ de la population autochtone vivant au Chili résiderait en milieu urbain. La dernière partie du mémoire met en lumière ces recompositions identitaires et territoriales. C'est là le dernier axe de lecture d'un territoire dont la géographie peut donc aussi être appréhendée à partir des territorialités vécues, celles des pratiques du quotidien.

Fiche Informative

Discipline

Géographie 
Directeur

Denis RETAILLE

Université

Université de Rouen

Membres du jury de thèse, soutenue le 17 juin 2011

- Denis RETAILLE, Professeur, Université de Bordeaux (directeur)

- Fernando CASANUEVA, Professeur, Université de Bordeaux (rapporteur)

- Caroline DESBIENS, Professeure agrégée, Université Laval, Québec (rapporteur)

- Odette LOUISET, Professeure, Université de Rouen (présidente)

- Guillaume BOCCARA, Chargé de Recherche, CNRS-CERMA, EHESS

- Jean-Paul DELER, Directeur de Recherche, CNRS-ADES, Bordeaux

Contact de l'auteur

bastien_sepulveda[at]yahoo.fr

\section{NOTES}

1. Suite au coup d'Etat du 11 septembre 1973, Augusto Pinochet instaura au Chili l'une des plus longues (1973-1989) et des plus sanglantes dictatures d'Amérique du Sud.

INDEX

Thèmes : Carnets de soutenances 